\title{
ON GROUPS EVERY CYCLIC SUBGROUP OF WHICH IS A POWER OF THE GROUP
}

\author{
By \\ F. SZÁSZ (Debrecen) \\ (Presented by L. RÉDEI) \\ To the memory of my beloved master T. SzelE.
}

\section{$\S 1$. Introduction}

In recent years many group-theoretical investigations originated with the purpose of giving an explicit characterization of some class of groups defined by some fixed property. The present paper is devoted to a similar problem.

Let $k$ be a fixed natural number not necessarily different from zero and let $G$ be an arbitrary not necessarily abelian group written multiplicatively. The $k$-th power of the group $G$ is a subgroup denoted by $G^{k}$, generated by the set of the $k$-th powers of all elements of $G$.

We shall call an arbitrary group $G$ a group with property $\mathrm{P}$, if to every cyclic subgroup $H$ of $G$ there exists a natural number $k$ for which $H=G_{k}$ holds. Every cyclic group is a group with property P. The aim of this paper is to give an elementary proof of the converse statement, namely we are going to prove that a group with property $\mathrm{P}$ is necessarily cyclic, i. e. property $\mathrm{P}$ is characteristic for cyclic groups.

\section{§ 2. Preliminaries}

The characterization to be given of cyclic groups will be more easy to survey, if we prove first of all three lemmas. Before this, let us make some terminological remarks.

We shall not assume the commutativity of the groups considered and therefore we use multiplicative notation throughout. We denote the order of the element $g$ by the symbol $O(g)$. A group is called torsion free if it contains no element of finite order other than the unity. On the other hand, groups every element of which is of finite order are called torsions groups. If a torsion group $G$ contains an element of maximal order $m$, then we say that $G$ is an m-bounded group. A group is termed a p-group if all its elements have orders which are powers of the fixed prime number $p$. A finite p-group always has a non-trivial center [2]. ${ }^{1}$ In any finite group $G$ of order $n=p_{1}^{\alpha_{1}} \ldots p_{i}^{\alpha_{i}} \ldots p_{r}^{\alpha_{r}}$ where $p_{1}, \ldots, p_{i}, \ldots, p_{r}$ are different prime numbers, there exists necessarily a $p_{i}$-subgroup of order $p_{i}^{\alpha_{i}}$, called a Sylow $p_{i}$-group of the group $G$ [2]. A group is called metabelian if its center contains its derived group, i. e., the subgroup generated by the commutators $[a, b]=a^{-1} b^{-1} a b$ of

1 The numbers in brackets refer to the Bibliography given at the end of this paper. 
all group elements $a$ and $b$. In metabelian groups the relation $[a, b c]=$ $=[a, b] \cdot[a, c]$ holds for arbitrary elements $a, b, c[2]$.

Now we prove the lemmas.

Lemma 1. A group with property $\mathrm{P}$ is a torsion free or a finite group.

PROOF. Let $G$ be an arbitrary not torsion free group with property $\mathrm{P}$, and let $g \in G$ where $O(g)=n>1$. If $\{g\}=G^{k}$, then $G$ is obviously an $m$ bounded group, where $m \leqq n k$. In this case the set of all different powers of $G$ is finite. Therefore the number of all cyclic subgroups of $G$ is also finite, hence the torsion group $G$ contains but a finite number of elements.

LEMma 2. Every homomorphic image $G^{\prime}$ of a group $G$ with property $\mathrm{P}$ is a group with property $\mathrm{P}$.

Proof. Let $G$ be an arbitrary group with property $\mathrm{P}$ and $\varphi$ a homomorphic mapping of $G$ onto the group $G^{\prime}$. If $H^{\prime}$ is an arbitrary cyclic subgroup generated by the element $h^{\prime}$ of $G^{\prime}$, then there exists an element $h$ of $G$ for which $h \varphi=h^{\prime}$ holds. Let $\{h\}=H=G^{k}$ and thus $h=g_{1}^{k} \cdots g_{s}^{k}$ with certain elements $g_{1}, \ldots, g_{s}$ of $G$. Obviously, we have $H \varphi=H^{\prime}$ and $h^{\prime}=$ $=\left(g_{1} \varphi\right)^{k} \cdots\left(g_{s} \varphi\right)^{k}$, whence $\left(G^{k}\right) \varphi=\left(G^{\prime}\right)^{k}$. On the other hand, if $g \in G$, then $\left(g^{k}\right) \varphi=(g \varphi)^{k} \in H \varphi$, therefore we can write $\left(G^{\prime}\right)^{k} \subseteq\left(G^{k}\right) \varphi$, and thus $H^{\prime}=\left(G^{\prime}\right)^{k}$.

Lemma 3. A finite group with property $\mathrm{P}$ has always a non-trivial center.

PROOF. Let $\varepsilon$ be an arbitrary endomorphism of the group $G$ with property $\mathrm{P}$ and $H$ a cyclic subgroup of $G$. Then, by the proof of Lemma 2, $\left(G^{n}\right) \varepsilon=(G \varepsilon)^{n}$ is valid for any natural number $n$, and thus $H=G^{k}$ implies $H \varepsilon=\left(G^{k}\right) \varepsilon=(G \varepsilon)^{k} \subset G^{k}=H$. But then every subgroup $S$ is also mapped into itself, becaușe $g \in S$ implies $\{g\} \subset S$ and $g \varepsilon \in\{g\} \varepsilon \subseteq\{g\} \subseteq S$ for every endomorphism $\varepsilon$ of the group $G$. Thus every Sylow p-group of a finite group $G$ is normal in $G$, and so $G$ is the direct product of its Sylow p-groups. Since finite p-group is never without center and the center of a direct product is the direct product of the centers of the direct factors, therefore $G$ has a non-trivial center.

\section{$\S 3$. A characterization of the cyclic groups}

Now we prove the following

THEOREM. An arbitrary group is cyclic if and only if it has property P.?

2 By our theorem it is clear that if a group $G$ has property $\mathrm{P}$ then to every subgroup $S$ of $G$ there exists a natural number $n$, for which $S=G^{n}$ holds. In a previous paper written in Hungarian with German summary (Über zyklische Gruppen, Acta Scientiarum Universitatis Debreceniensis de Ludovico Kossuth nominatae) I have proved this theorem in the following weaker form: if for every subgroup $S$ of a group $G$ there exists a natural number $n$ for which $S=G^{n}$ holds, then $G$ is necessarily cyclic. The proof of this weaker theorem made use of the fundamental theorem of R. BAER on the structure of hamiltonian groups [1]. 
Proof. Let first $G$ be an arbitrary finite group with property $\mathrm{P}$ and let $m$ be the order of $G$. In this case we prove our statement by induction on $m$. The theorem is trivially true for $m=1$, and we assume that it holds for all finite groups with property $\mathrm{P}$ of order $s$ less than $n$. By Lemma 3 the center $C$ of $G$ differs from $\{1\}$. By our induction hypothesis the factor group $G / C$ is cyclic, because it is by Lemma 2 a group with property $\mathrm{P}$ and on the other hand the order of $G / C$ is $<m$. Considering that the factor group with respect to the center is never cyclic, we conclude that $G$ is a finite abelian group with property P. By the fundamental theorem on finite abelian groups there exists in every Sylow p-group $S_{p} \neq\{1\}$ of $G$ a cyclic direct factor $A_{p} \neq\{1\}$ of $S_{p}$, so that $S_{p}=A_{p} \times B_{p}$ with a certain subgroup $B_{p}$ of $S_{p}$. But $A_{p}=G^{k}$ and $G=A_{p} \times B_{p} \times M$ imply $A_{p}=A_{p}^{k} \times B_{p}^{k} \times M^{k}$. Here we must have $B_{p}^{k}=M^{k}=\{1\}$ and $A_{p}=A_{p}^{k}$, so that $(k, p)=1$. Then $B_{p}=\{1\}$, and thus each $p$-component of $G$ being cyclic, $G$ itself is a finite cyclic group.

If $G$ is infinite, then, by Lemma 1 , the group $G$ with property $\mathrm{P}$ is necessarily torsion free. Let now $a$ and $b$ be arbitrary elements of $G$. The subgroup $T=\{a, b\}$ is metabelian, because by the proof of Lemma 3 every subgroup of $G$ is normal, therefore the commutator $c=[a, b]=a^{-1} b^{-1} a b$ is contained in the intersection $\{a\} \cap\{b\}$, and so in the center of the subgroup $T$ of $G$. If $c=a^{s}=b^{t}$, then $c^{s t}=[a, b]^{s t}=\left[a^{s}, b^{t}\right]=[c, c]=1$ and thus $[a, b]=1$. Therefore $G$ is an abelian torsion free group. If $n \neq 0$, then the mapping $\varphi$ of $G$ into itself, where $g_{r p}=g^{n}$, is an isomorphism. Let $g \in G$ and $g \neq 1$. If $\{g\}=G^{k}$, then $G$ itself is necessarily cyclic.

On the other hand, every cyclic group has obviously the property P; this completes the proof of our Theorem.

\section{(Received 4 July 1955)}

\section{Bibliography}

[1] R. BAER, Situation der Untergruppen und Struktur der Gruppe, S. B. Heidelberg. Akad., 2 (1933), pp. 12-17.

[2] А. Г. Кур ош, Теория групп (Москва, 1953).

\section{О ГРУППАХ, КАЖДАЯ ЦИКЛИЧЕСКАЯ ПОДГРУППА КОТОРЫХ ЯВЛЯЕТСЯ} НЕКОТОРОЙ СТЕПЕНЬЮ ГРУППЫ

$$
\begin{gathered}
\text { Ф. С а с (Дебрецен) } \\
\text { (Р е з ю м е) }
\end{gathered}
$$

Произвольная группа $G$ называется группой со свойством Р, если каждая циклическая подгруппа $H$ группы $G$ является некоторой степенью $G^{k}$ группы $G$, где $k$-й степень $G^{k}$ группы $G$ есть подгруппа, порожденная множеством $k$-ых степеней всех элементов группы $G$. Әлементарным способом доказывается, что произвольная группа $G$ является циклической тогда и только тогда, если она имеет свойство Р. 